\title{
De Haas-van Alphen effect and energy gaps of a correlated two-dimensional electron system in an AlAs two-valley pseudospin system
}

\author{
T. Windisch, ${ }^{1}$ X. Huang, ${ }^{1}$ S. Dasgupta, ${ }^{2}$ B. Rupprecht, ${ }^{1}$ Ch. Heyn, ${ }^{3}$ M. Bichler, ${ }^{2}$ A. Fontcuberta i Morral,,${ }^{2,4}$ M. Grayson, ${ }^{5}$ \\ G. Abstreiter, ${ }^{2}$ M. A. Wilde, ${ }^{1, *}$ and D. Grundler ${ }^{1}$ \\ ${ }^{1}$ Physik Department, Lehrstuhl für Physik funktionaler Schichtsysteme, Technische Universität München, James-Franck-Str. 1, D-85747 \\ Garching b. München, Germany \\ ${ }^{2}$ Walter Schottky Institut, Technische Universität München, Am Coulombwall 3, D-85748 Garching b. München, Germany \\ ${ }^{3}$ Institut für Angewandte Physik und Zentrum für Mikrostrukturforschung, Universität Hamburg, D-20355 Hamburg, Germany \\ ${ }^{4}$ Laboratoire des Matériaux Semiconducteurs, Institut des Matériaux, Ecole Polytechnique Féderale de Lausanne, \\ 1015 Lausanne, Switzerland \\ ${ }^{5}$ McCormick Engineering, EECS, Northwestern University, Evanston, Illinois 60208-3118, USA \\ (Received 25 August 2009; revised manuscript received 13 October 2009; published 9 November 2009)
}

\begin{abstract}
We report highly sensitive de Haas-van Alphen (dHvA) effect measurements on a high-mobility twodimensional electron system in an AlAs quantum well. Here two valleys are occupied forming a pseudospin system. At $400 \mathrm{mK}$, the dHvA effect shows pronounced oscillations at filling factors $\nu=1$ to four. In the quantum limit at $\nu=1$ the data are consistent with an interaction-enhanced valley splitting, which exceeds the Zeeman spin splitting in a perpendicular field $B$. When tilting $B$ the energy gap $\Delta E$ at $\nu=1$ shows first an unexpectedly strong angular dependence and then remains constant. This suggests a crossover in the energy gap, most likely from a spin to a pseudospin gap. We attribute the strong initial dependence of $\Delta E$ on the tilt angle to skyrmion-type spin excitations. Surprisingly, the dHvA oscillation amplitudes do not display coincidence phenomena at higher filling factors. This is explained by the large valley splitting and avoided crossings of energy levels.
\end{abstract}

DOI: 10.1103/PhysRevB.80.205306

PACS number(s): 73.21.-b, 71.70.Di, 73.40.Kp, 73.43.Fj

\section{INTRODUCTION}

Two-dimensional electron systems (2DESs) in AlAs quantum wells (QWs) have shown novel and intriguing phenomena ${ }^{1}$ such as a strongly enhanced spin susceptibility ${ }^{2}$ and quantum Hall "valley skyrmions". ${ }^{3}$ In these QWs the 2DES can be twofold valley degenerate with a heavy anisotropic mass. The parameter $r_{s}$ (defined as the average interelectron spacing in units of the effective Bohr radius) is large. This makes such a 2DES in AlAs an interesting strongly interacting electron system, which exhibits an additional pseudospin degree of freedom due to the two occupied valleys. To explore interaction-induced phenomena in further detail, measurements of the magnetization $\mathbf{M}$ can improve upon coincidence magnetotransport experiments ${ }^{1}$ by giving direct access to the 2DES's ground-state energy $U$. As a thermodynamic quantity, the magnetization $M=-\partial U /\left.\partial B\right|_{n_{s}, T=0}$ provides information about the energy levels in a magnetic field $B\left(n_{s}\right.$ is the electron density). The quantum oscillations $M(B)$ of the de Haas-van Alphen (dHvA) effect reflect, in particular, energy gaps of the many-body system. ${ }^{4}$ However, studies on AlAs are an experimental challenge since the effective electron cyclotron mass $m_{c}^{*}$ is large. Following Ref. 5, this makes the dHvA amplitude $\Delta M$ hard to detect. However, after initial experiments on 2DESs had been reported in Ref. 6 , there have been important developments to improve the resolution of dHvA studies. ${ }^{7}$ Improvements were achieved by either sophisticated torque magnetometers using torsion balance along a thin wire ${ }^{8-10}$ or a specially designed superconducting quantum interference device (SQUID) at $300 \mathrm{mK}$ using very low-noise SQUID electronics. ${ }^{11,12}$ These approaches provided a high sensitivity but did not allow to study the dHvA effect under a large tilt angle. This was realized recently using a micromechanical torque magnetometer. ${ }^{13}$ References 14 and 15 provide a review on the status in this field.

In Ref. $13 \mathrm{dHvA}$ measurements were performed on a 2DES in a strained $\mathrm{Si} / \mathrm{SiGe}$ heterostructure where $m_{c}^{*}$ $=0.19 m_{e}$ was already relatively large $\left(m_{e}\right.$ is the free-electron mass). In $\mathrm{Si} / \mathrm{SiGe}$ the $2 \mathrm{DES}$ occupied two valleys, which were isotropic and concentric in momentum space. Pronounced coincidence phenomena were found in tilted magnetic fields $\mathbf{B}$ because energy gaps resulting from Landau quantization $\left(\Delta E_{L}\right)$, spin splitting $\left(\Delta E_{S}\right)$, and field-induced lifting of valley degeneracy $\left(\Delta E_{V}\right)$ were tuned to the same value. The tilted-field-induced level crossings produced a clear signature, i.e., $\Delta M=0$, for a given filling factor $\nu$ $=n_{s} h /\left(e B_{\perp}\right)$ at a specific tilt angle $\alpha\left(B_{\perp}=B \cos \alpha\right)$.

In this paper, we report magnetic quantum oscillations of a $2 \mathrm{DES}$ in an AlAs QW with a twofold valley degeneracy at zero $B$. Here, the anisotropic valleys are separated in momentum space by half an umklapp vector. Surprisingly, we find a strong increase in the $\nu=1$ energy gap with increasing $\alpha$. At the same time we do not observe coincidences, i.e., zeros in $\Delta M$, at higher $\nu$. Our findings suggest that electronelectron interaction is strong and leads to both correlated states with large spin-flip number $\Delta S$ and an avoided crossing of energy levels.

The paper is organized as follows. We discuss details of the AlAs quantum well and the experimental setup in Sec. II and summarize the experimental results in Sec. III. We analyze and discuss the data in Sec. IV and conclude with Sec. V. 


\section{EXPERIMENT}

The measurements were performed on a 2DES, which resided in a 15-nm-wide AlAs QW embedded in barrier layers of $\mathrm{Al}_{0.45} \mathrm{Ga}_{0.55} \mathrm{As}$. The layer sequence was grown on an (001) GaAs substrate. Due to biaxial strain between the AlAs QW and the $\mathrm{Al}_{0.45} \mathrm{Ga}_{0.55}$ As layers, the two in-plane valleys are lowered in energy by $\approx 16 \mathrm{meV}$ with respect to the outof-plane valley and occupied by electrons. The effective electron mass is anisotropic with $m_{l}=1.1 m_{e}$ and $m_{t}=0.2 m_{e}{ }^{16}$ For our dHvA experiment the cyclotron mass is thus $m_{c}^{*}$ $=\sqrt{m_{l} m_{t}}=0.47 m_{e}$, which is a factor of 2.5 larger than in Si/ SiGe. This makes a high electron mobility $\mu$ necessary in order to reduce disorder broadening of the Landau levels to the point where magnetization is measurable. To this end, we optimized the modulation doping structure in such a manner that we reached $\mu=4.2 \times 10^{5} \mathrm{~cm}^{2} / \mathrm{Vs}$, exceeding previously published mobilities at the same density $n_{s}$ by about a factor of seven. The sample was illuminated using a protocol described in detail elsewhere. ${ }^{17-19}$ We measured $\mu$ on a reference sample in van-der-Pauw configuration at $300 \mathrm{mK}$. The band-structure $g$ factor was $g=2 .{ }^{20}$

We used a micromechanical cantilever magnetometer to measure the anisotropic magnetization $\mathbf{M}$ of the 2DES. The sensor was micromachined from undoped $\mathrm{AlGaAs} / \mathrm{GaAs}$ layers grown by molecular-beam epitaxy similar to the procedure described in Ref. 21. The 2DES had an area of 0.8 $\times 1.6 \mathrm{~mm}^{2}$ and resided in a separate $20 \mu \mathrm{m}$-thick sample piece that was glued to the end of the flexible cantilever beam. The electron density $n_{s}$ and parameter $r_{s}$ (derived from $\left.m_{c}^{*}\right)$ were $2.4 \times 10^{11} \mathrm{~cm}^{-2}$ and 10.2 , respectively. We measured $\mathbf{M}$ via the torque $\boldsymbol{\tau}=\mathbf{M} \times \mathbf{B}$. The resulting deflection of the cantilever was detected using a capacitive readout. ${ }^{22}$ To tilt the 2DES normal with respect to $\mathbf{B}$ by an angle $\alpha$ we mounted the cantilever on a Swedish rotator. ${ }^{23} B_{\perp}$ is the field component perpendicular to the 2DES. In the experiment $B_{\|}=B \sin \alpha$ points symmetrically to the principal axes of the in-plane conduction-band valleys [see Fig. 1(d)]. By using this geometry we intentionally avoid the recently reported $B_{\|}$-induced asymmetry between the two valleys. ${ }^{24}$

\section{EXPERIMENTAL RESULTS}

In Figs. 1(a) and 1(b) we show the oscillatory part of $M(B)$ for $\alpha=55^{\circ}$ and $\alpha=40^{\circ}$, respectively. The data are obtained at $400 \mathrm{mK}$. A smooth magnetic background has been removed from the raw data by subtracting a low-order polynomial fit in $1 / B \cdot{ }^{10,25,26}$ We find a sawtoothlike dHvA signal whose sharply dropping slopes coincide with the integer filling factors. The peak-to-peak amplitude at filling factor $\nu$ $=2$ in panel (a) amounts to $\Delta M_{\nu=2}=3.6 \times 10^{-14} \mathrm{~J} / \mathrm{T}$, one order of magnitude smaller than in $\mathrm{Si} / \mathrm{SiGe}^{22}$ The amplitude per electron amounts to $\Delta M_{e, \nu=2}=0.76 \mu_{B}^{*}$, where $\mu_{B}^{*}$ $=\mu_{B}\left(m_{e} / m_{c}^{*}\right)=1.97 \times 10^{-23} \mathrm{~J} / \mathrm{T}\left(\mu_{B}\right.$ is the Bohr magneton $)$. This value $\Delta M_{e, \nu=2}$ for the 2DES in AlAs is $25 \%$ larger than the corresponding value in $\mathrm{Si} / \mathrm{SiGe}$. This underscores both the small broadening of the Landau levels and the high quality of this AlAs electron system. Further magnetization traces such as those shown in Fig. 1(c) have been similarly analyzed.

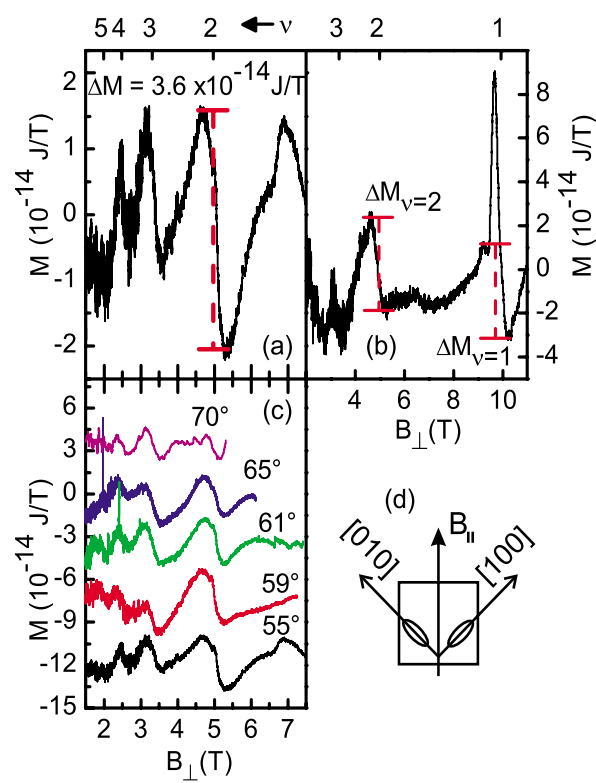

FIG. 1. (Color online) (a) Oscillatory part of the magnetic signal detected by the micromechanical sensor at $\alpha=55^{\circ}$ and $T=400 \mathrm{mK}$ ( $\sim 45 \mathrm{~s}$ per data point, $0.1 \mathrm{~T} / \mathrm{min}$ sweep). The dashed line highlights the peak-to-peak amplitude $\Delta M$ of the dHvA effect. (b) Oscillations at $\alpha=40^{\circ}$ in the quantum limit. The spike at $\nu=1$ is due to an eddy current. This is a known nonequilibrium effect, which appears in a high-mobility 2DES. This signal is parasitic and not considered in evaluating the dHvA oscillation amplitude (see text). (c) dHvA effect for different tilt angles $\alpha$. Curves are offset for clarity. (d) Orientation of $\mathbf{B}_{\|}$with respect to the occupied inplane valleys.

We note that in Fig. 1(b) there is an additional spike occurring at $\nu=1$, which is not noise but reflects a so-called eddy current. This is a well-known nonequilibrium effect. Eddy currents in $M$ have been investigated in detail previously and are found to occur if the longitudinal resistivity of a 2DES vanishes, i.e., if energy levels in a quantizing magnetic field are well separated and the energy gaps have formed. ${ }^{14,27}$ Our observation of an eddy current at $\nu=1$ indicates that here the levels do not overlap significantly. This will become important for the discussion. The eddy-current signal can be clearly distinguished from the equilibrium dHvA effect because eddy currents flip sign if one changes the sweep direction. In contrast to this, the dHvA effect is a thermodynamic equilibrium phenomenon and does not depend on the sweep direction. Eddy currents are interesting by themselves (see Ref. 14) but will not be investigated further in this paper.

In Fig. 2(a) we summarize dHvA amplitudes $\Delta M_{\nu}$ for different filling factors $\nu$ and different tilt angles $\alpha$. An important finding is that $\Delta M_{\nu=1}$ (filled squares) increases significantly with $\alpha$ between $15^{\circ}$ and $30^{\circ}$, becoming larger than $\Delta M_{\nu=2}$ (open squares). Such a behavior has not been reported for the dHvA effect of a 2DES before. ${ }^{4,8-10,14}$

\section{ANALYSIS AND DISCUSSION}

\section{A. Data analysis}

From $\Delta E_{\nu}=\Delta M_{e, \nu} B_{\perp, \nu}$ we calculate energy gaps $\Delta E$ of the electronic spectrum at integer filling factors $\left(B_{\perp, \nu}\right.$ is the 


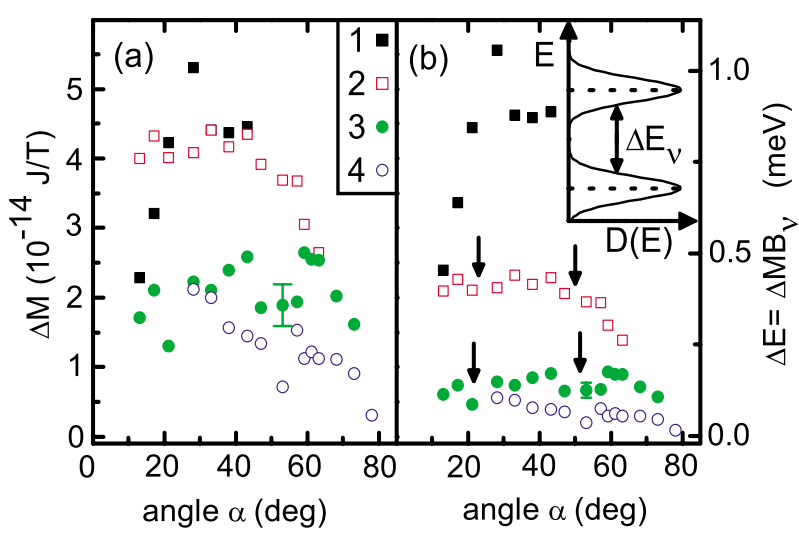

FIG. 2. (Color online) (a) Angular dependence of the dHvA amplitude $\Delta M$ for different filling factors $\nu . \Delta M$ of $\nu=1$ increases by about a factor of two between $15^{\circ}$ and $30^{\circ}$, whereas $\Delta M$ of $\nu=2$ does not vary much in this regime. Numbers labeling symbols in the inset denote filling factors. (b) Energy gaps $\Delta E_{\nu}$ calculated from the data in (a). Arrows indicate local minima for $\nu=2,3$, and 4. The inset illustrates that the energy gap $\Delta E_{\nu}$ is reduced by level broadening. Error bars are indicated in (a) and (b).

magnetic-field position of the corresponding filling factor). ${ }^{9,28,29}$ These gaps are called thermodynamic energy gaps to distinguish them on the one hand from gaps obtained by excitation spectroscopy where selection rules must be considered. Rules such as the generalized Kohn's theorem might obscure electron-electron interaction effects. ${ }^{30}$ They do not apply to the dHvA effect, where the amplitude reflects all many-body effects. On the other hand this term highlights that the dHvA effect monitors energy gaps in the groundstate electronic spectrum. The values are displayed in Fig. 2(b). It is striking that $\Delta E_{\nu=1}$ is by far the largest and constant after a strong initial increase at small tilt angles. The increase is unexpected. In particular, it is not consistent with previous magnetotransport data on an AlAs QW of the same width and density $n_{s}$ but a smaller mobility $\mu .{ }^{31}$ At $\nu=1$ those authors reported a monotonically decreasing energy gap when increasing $\alpha$ from 0 to $50^{\circ}$. We find a different behavior. We suggest that the higher mobility of our 2DES makes electron-electron interaction effects more prominent than previously measured.

The dHvA effect provides values for the energy gaps $\Delta E$ at $\nu=3$ and $\nu=4$ over a broad angular regime, i.e., from $15^{\circ}$ to $80^{\circ}$. Interestingly these gaps are always nonzero and no coincidence signature with $\Delta M=0$ is observed. The same is true for $\nu=2$ up to $65^{\circ}$. Instead we find small variations in $\Delta E_{\nu}$ with $\alpha$ (marked by arrows) at filling factors $\nu \geq 2$. The absence of coincidences in Fig. 2(b) contrasts with the case of $\mathrm{Si} / \mathrm{SiGe} .{ }^{13}$ In the following we interpret our results in terms of interaction effects in the high-mobility 2DES.

\section{B. Level structure}

In an ideal 2DES a discontinuous jump $\Delta M$ occurs in the dHvA effect whenever the Fermi level crosses an energy gap. In our AlAs sample we consider the same situation as in $\mathrm{Si} / \mathrm{SiGe}$ of Ref. 13, in that we assume that energy gaps are induced by Landau quantization, Zeeman spin splitting, and
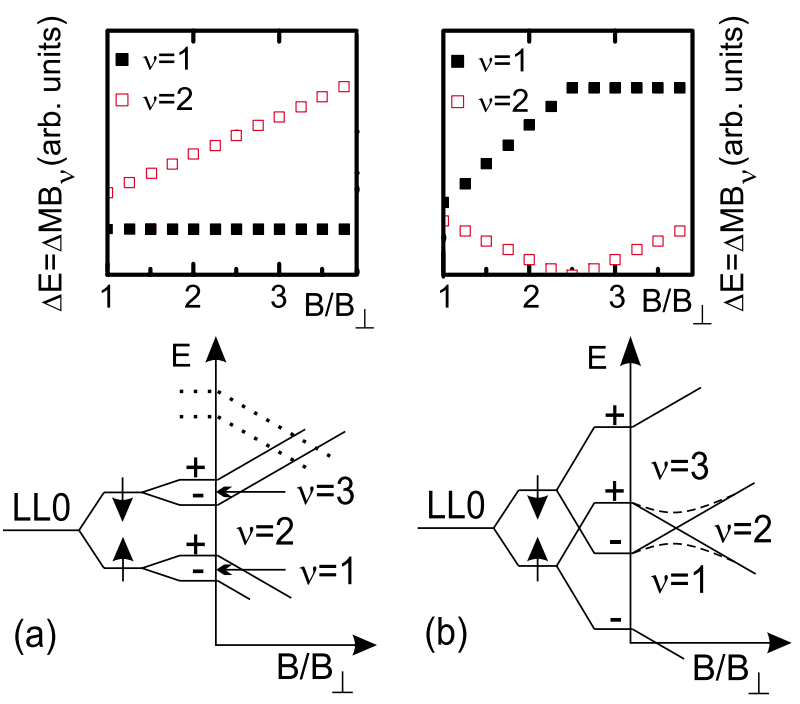

FIG. 3. (Color online) Two different scenarios (level sequences) for the splitting of the lowest Landau level (LL0): (a) $\Delta E_{V}<\Delta E_{S}$ and (b) $\Delta E_{V}>\Delta E_{S}$. Labels $(\uparrow, \downarrow)$ and $(+,-)$ indicate spin and valley quantum numbers, respectively. The insets display the evolution of energy gaps $\Delta E_{\nu}=\Delta M_{\nu} B_{\perp, \nu}$ as a function of $\alpha$ for $\nu=1$ and 2. In the schematics, possible exchange enhancement effects are assumed to be independent of $B / B_{\perp}$.

the magnetic-field-induced lifting of the valley degeneracy. We describe the level structure using the Landau-level index $n$, spin index $(\uparrow, \downarrow)$, and valley index $(+,-)$ (Fig. 3). A priori it is not clear which of the gaps is the largest in a high magnetic field $B$. In Figs. 3(a) and 3(b) we display schematically two different scenarios for the lowest Landau level LL0, where we consider a small and a large valley splitting $\Delta E_{V}$, respectively. Increasing the tilt angle $\alpha$ shifts the energetic position of the levels due to an increased spin splitting $\Delta E_{S}$. The spin splitting is dependent on the total field $B$, whereas the Landau quantization depends only on the perpendicular component $B_{\perp}$. Valley splitting has also been found to depend only on $B_{\perp} \cdot{ }^{13}$ For filling factors $\nu=1$ and $\nu=2$ we can distinguish two extreme cases in Fig. 3: in (a) no level crossing occurs in the quantum limit for increasing $\alpha$ since $\Delta E_{V}<\Delta E_{S}$. Here, $\Delta E_{\nu=1}$ is expected to stay constant, since the $\nu=1$ gap is always between the different valley states $(0, \uparrow,-)$ and $(0, \uparrow,+)$ of the same spin orientation. Energetically, these levels move in parallel when $\alpha$ is varied. $\Delta E_{\nu=2}$ is expected to increase with $\alpha$ [Fig. 3(a), inset]. In (b), however, $\Delta E_{V}>\Delta E_{S}$ so that $\Delta E_{\nu=1}$ first increases with $\alpha$ and only later stays constant after a level crossing between levels $(0, \uparrow,+)$ and $(0, \downarrow,-)$. The gap $\Delta E_{\nu=2}$ first reduces to zero and then increases again with increasing $\alpha$ [Fig. 3(b), inset]. In this model, a value $\Delta E_{\nu=2}=0$ would be expected at a specific angle $\alpha$.

On the basis of Fig. 3(b), our dHvA data and the characteristic behavior of $\Delta E_{\nu=1}$ suggest that the level sequence exhibits a large valley splitting $\Delta E_{V}>\Delta E_{S}$ when $\alpha=0$, i.e., $B / B_{\perp}=1 / \cos \alpha=1$. The level sequence (b) can, in particular, explain the characteristic increase in $\Delta E_{\nu=1}$ with $\alpha$ followed by a constant value. For higher filling factors $\nu$ both scenarios predict coincidences, i.e., $\Delta M=0$ for specific values of $\alpha$. In scenario (a) tilted-field-induced coincidences are ex- 
pected to occur for $\nu \geq 3$, in scenario (b) for $\nu \geq 2$. In both scenarios the maximum angle for a coincidence would be given by $\alpha=\arccos \left(g m_{c}^{*} / 2 m_{e}\right)=62^{\circ}$. Importantly, we do not observe $\Delta M=0$ for $15^{\circ}<\alpha<75^{\circ}$. This might indicate that the tilted field does not provoke a crossing of levels, but instead the levels might be coupled strongly and anticross. ${ }^{32}$ Then the levels would follow the broken lines in Fig. 3(b). Consistent with such avoided crossings our data in Fig. 2(b) show small variations in $\Delta M_{\nu} B_{\perp, \nu}$ for $\nu \geq 2$ leading to local minima (marked by arrows) as a function of $\alpha$.

At this point we rule out that coupling to the higher bands is relevant for the findings discussed above. We calculated the specific energy-level structure of the 15-nm-wide AlAs quantum well using the simulation code nextnano ${ }^{3}$ provided by Ref. 33. We obtained a level spacing of 16.4 (17.5) meV between the occupied subband and the lowest subband of the out-of-plane valley (next higher subband of the in-plane valleys). Taking $m_{c}^{*}=0.47 m_{e}$ as relevant for the density-of-states mass and considering that two in-plane valleys are occupied we calculate a Fermi energy of $E_{F}=0.6 \mathrm{meV}$ from the carrier density $n_{s}$. The next higher lying subband is thus 15.8 meV apart. This suggests that coupling to higher bands has a minor impact on the Landau levels considered here. In Ref. 23 it was shown that coupling to a second subband leads to both a warping of the Landau levels and a smooth decrease in $\Delta M_{\nu}$ and $\Delta E_{\nu}$ at high tilt angles. The experimental data in Figs. 2(a) and 2(b) show a slight decrease at large $\alpha$. However, the scenario of Ref. 23 does not explain the absence of coincidences and the strong variation in the energy gap at $\nu=1$ at small tilt angles.

\section{Energy gap at $\nu=1$}

In the following, we analyze the characteristic behavior of $\Delta E_{\nu=1}$ in detail. For $\nu=1$ and small $\alpha\left(B / B_{\perp}\right), E_{F}$ is assumed to be between levels $(0, \downarrow,-)$ and $(0, \uparrow,-)$ [Fig. 3(b)]. These states differ in the spin but not the valley quantum number. Following Refs. 13 and 34 we can evaluate this energy gap due to spin splitting by using

$$
\Delta E_{\nu=1}=\Delta S g \mu_{B} B+\gamma E_{C},
$$

where $E_{C}=e^{2} / 4 \pi \epsilon \epsilon_{0} l_{B}$ is the Coulomb energy $\left(l_{B}^{2}=e B_{\perp} / h\right.$ is the magnetic length, $\epsilon$ is the dielectric constant, and $\gamma$ is a prefactor considering relevant matrix elements and the difference in population of the spin-up and spin-down levels). This equation models the angular dependence and is a generalization, to the case $\Delta S>1$, of the result given by Ando and Uemura $^{35}$ for the energy associated with a spin flip in a 2DES formed in the inversion layer of a $\mathrm{Si}$ metal-oxidesemiconductor device. The first term is the Zeeman spin splitting energy $\Delta E_{Z}$, which is assumed to be the only quantity that depends on the absolute field $B$, i.e., the $2 \mathrm{DES}$ is taken as infinitely thin. In this case, an in-plane magneticfield component couples to the system only through the Zeeman energy. The perpendicular magnetic-field component governs the orbital dynamics. ${ }^{34}$ The second term models the electron-electron interaction, which leads to an exchange enhancement of the spin splitting and is known to depend on $B_{\perp}$ via the Coulomb energy $E_{C} \cdot{ }^{35}$ This term might also in-

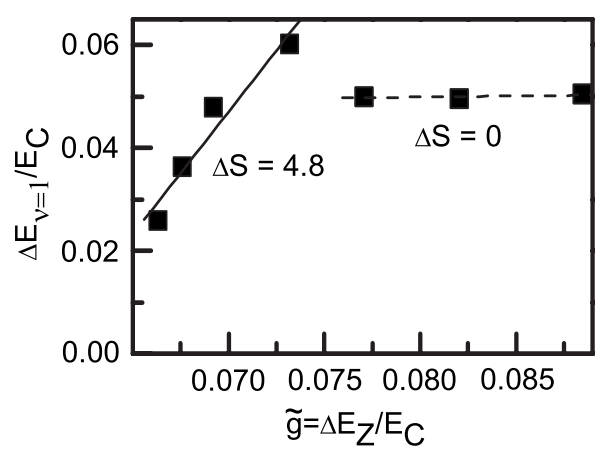

FIG. 4. Energy gap $\Delta E_{\nu=1}$ (symbols) plotted as a function of Zeeman energy $\Delta E_{Z}$, both normalized to the Coulomb energy $E_{C}$. The steep slope corresponds to the spin-flip number per charge excitation $\Delta S=4.8 \pm 0.9$ (solid line). At large tilt angle we observe a constant value reflecting $\Delta S=0 \pm 0.05$ (dashed line).

clude a difference in the valley splitting of the lower and upper spin branch, which also depends only on $B_{\perp} . \Delta S$ is the fitting parameter. In Fig. 4 we replot the measured energy gaps $\Delta E_{\nu=1}$ normalized to the Coulomb energy versus the normalized Zeeman energy $\tilde{g}=g \mu_{B} B / E_{C}$. We find two different regimes: at small $\tilde{g}=g \mu_{B} B / E_{C}$ we find a steep slope giving $\Delta S=4.8 \pm 0.9$. This value is taken from the least-squares fit to our data at $\alpha<30^{\circ}$. The data at higher tilt angles correspond to $\Delta S=0 \pm 0.05$. Both values are strikingly different from $\Delta S=1$, which is expected for spin-flip excitations of noninteracting electrons with $g=2$. A value $\Delta S \approx 1$ was found in the two-valley $\mathrm{Si} / \mathrm{SiGe}$ system for large filling factors. ${ }^{13}$ In Ref. 34 Schmeller et al. argued that $\Delta S \gg 1$ indicated correlated excitations, which were predicted to be the lowest energy excitations around $\nu=1$. The physical meaning of $\Delta S$ is the so-called skyrmion size, i.e., the number of correlated spins taking part in the excitation.

It is instructive to revisit the discussion given in Ref. 34 to substantiate the data analysis based on Eq. (1). The assumption that the in-plane magnetic-field component $B_{\|}$couples only through the Zeeman energy is only valid for an ideal, infinitely thin 2DES. In the experiment the real system exhibits a finite thickness, i.e., the magnetic confinement induced by $B_{\|}$and the electrostatic confinement of the heterostructure can hybridize. However, the energetic separation between subbands has been calculated to be large, so that we do not expect a strong effect of this hybridization.

The prefactor $\gamma$ in Eq. (1) contains both matrix elements and the relative population difference of the spin-up and spin-down levels, i.e., the degree of spin polarization. For a fixed filling factor, i.e., $\nu=1$ in our case, the matrix elements are constant and do not vary as a function of $B_{\|}$. The relative population difference in the spin-up and spin-down levels should take the value of one at $\nu=1$, i.e., the spin polarization should be at maximum if spin levels are well separated. Only when spin levels are broadened by disorder and overlap, the population difference varies as a function of $B_{\|}$. Indeed, it has been argued before that incomplete spin polarization due to level overlap might lead to the observation of a large slope $\Delta S .{ }^{36}$ If this disorder-driven mechanism was relevant for the large $\Delta S$ observed at $\nu=1$, we would have expected even larger slopes at larger spin filling factors, i.e., 
at smaller magnetic fields, where the energy gaps are smaller than at $\nu=1$. These further large slopes are not observed in the experiment. In particular, we find an eddy current at $\nu$ $=1$, which is induced by sweeping the magnetic field up or down (cf. Fig. 1). Importantly, this feature indicates that in our sample the spin-split levels do not overlap significantly as outlined above. We thus assume that the degree of spin polarization is high (close to one) already at small $B_{\|}$. As a consequence we argue that neither the finite thickness nor disorder effects are likely to explain the observed large initial value of $\Delta S$ at $\nu=1$. Instead, our results are in qualitative agreement with the behavior expected for skyrmionic excitations. In the literature, a large $\Delta S \approx 7$ was found for a singlevalley $2 \mathrm{DES}$ and small values of the Zeeman energy as compared to the Coulomb energy, i.e., $\widetilde{g} \leq 0.02 .{ }^{34}$ In contrast we find $\Delta S \approx 5 \gg 1$ near $\tilde{g}=0.07$. Skyrmions as the lowest lying excitations are predicted to be extremely sensitive to the details of the electronic structure. For our 2DES with both spin and pseudospin degrees of freedom, the relevant regime of $\widetilde{g}$ has not yet been predicted. A theoretical treatment, which considers large spin charge excitations for the magnetization or dHvA effect is lacking. Our observation is also different from the quantum Hall valley skyrmions at $\nu=1$ introduced by Shkolnikov et al. ${ }^{3}$ in an AlAs double-valley 2DES. They used in-plane strain to open and enlarge the gap $\Delta E_{\nu=1}$. This strain-induced gap did not depend on the tilt angle $\alpha$.

In contrast to Ref. 34, where the authors observed a smooth transition to a slope of $\Delta S=1$ at large $\tilde{g}$ we observe an abrupt change from $\Delta S \approx 5$ to $\Delta S=0$ at $\widetilde{g} \approx 0.075$ in Fig. 4. This substantiates that beyond $\tilde{g} \approx 0.075$ the nature of the energy gap changes in our case. Following scenario (b) in Fig. 3 at high $B / B_{\perp}$ the gap sits between two levels with the same spin but with a different valley quantum number. This is a pseudospin gap and real spin flips are no longer the relevant lowest lying excitations. The gap does not depend on $B_{\|}$, and $\Delta S=0$ becomes valid. In this regime we find $\Delta E_{\nu=1}=0.8 \mathrm{meV}$ from Fig. 2(b). We attribute this large value for the valley splitting to exchange enhancement.

\section{Energy gaps at $\boldsymbol{\nu} \geq \mathbf{2}$}

In the following we discuss the behavior of filling factors $\nu \geq 2$. The absence of coincidences suggests avoided level crossings. These can originate either from single-particle or many-body effects. In a single-particle picture, spin-orbit interaction (SOI) might cause the coupling. SOI, however, is assumed to be small for electrons in the conduction band of AlAs because the band gap is large and the band-structure $g$ factor is similar to the value $g=2$ of the free electron. For a many-body system one would expect Coulomb interaction to cause the strong coupling of levels. Within the framework of the Hartree-Fock approximation one might distinguish between direct Coulomb and exchange interaction as well as the correlation effects. At this point we are not able to decide which of these contributions is most effective in our highmobility 2DES in AlAs. Importantly, our data suggest avoided crossings to occur not only for $\nu=2$ but also for higher-indexed Landau levels. This must be considered by a many-body theory for pseudospin systems.

\section{CONCLUSION}

To conclude we reported the dHvA effect of a 2DES in an AlAs QW where two valleys were occupied. Both, the highly sensitive torque magnetometer and large mobility of $\mu=4.2$ $\times 10^{5} \mathrm{~cm}^{2} /$ Vs allowed us to monitor filling factors $\nu=1$ to four over a broad regime of tilt angles $\alpha$. The energy gap at $\nu=1$ showed a characteristic crossover behavior with a strong angular dependence reminiscent of skyrmionic spin excitations previously observed in single-valley systems. The strong dependence was found for the spin but not for the pseudospin degree of freedom. Our findings in the quantum limit suggest that many-body effects dominate the dHvA oscillations of the AlAs QW.

\section{ACKNOWLEDGMENTS}

We thank S. Birner for theoretical support. Financial support by the BMBF via nanoQUIT, Project No. 01BM470 and by the DFG via the Excellence Cluster "Nanosystems Initiative Munich (NIM)" as well as GR1640/3 in SPP 1285 "Halbleiter-Spintronik" and NSF CAREER Award DMR0748856 is gratefully acknowledged. *mwilde@ph.tum.de

${ }^{1}$ M. Shayegan, E. P. De Poortere, O. Gunawan, Y. P. Shkolnikov, E. Tutuc, and K. Vakili, Phys. Status Solidi B 243, 3629 (2006).

${ }^{2}$ K. Vakili, Y. P. Shkolnikov, E. Tutuc, E. P. De Poortere, and M. Shayegan, Phys. Rev. Lett. 92, 226401 (2004).

${ }^{3}$ Y. P. Shkolnikov, S. Misra, N. C. Bishop, E. P. De Poortere, and M. Shayegan, Phys. Rev. Lett. 95, 066809 (2005).

${ }^{4}$ I. Meinel, T. Hengstmann, D. Grundler, D. Heitmann, W. Wegscheider, and M. Bichler, Phys. Rev. Lett. 82, 819 (1999).

${ }^{5}$ D. Shoenberg, Magnetic Oscillations in Metals (Cambridge University, Cambridge, England, 1984).

${ }^{6}$ F. F. Fang and P. J. Stiles, Phys. Rev. B 28, 6992 (1983).

${ }^{7}$ Fang and Stiles measured the susceptibility of a 2DES in Ref. 6 using an inductive technique and concluded that they were not able to resolve the magnetization jumps associated with the dHvA effect.

${ }^{8}$ J. P. Eisenstein, H. L. Stormer, V. Narayanamurti, A. Y. Cho, A. C. Gossard, and C. W. Tu, Phys. Rev. Lett. 55, 875 (1985).

${ }^{9}$ S. A. J. Wiegers, M. Specht, L. P. Lévy, M. Y. Simmons, D. A. Ritchie, A. Cavanna, B. Etienne, G. Martinez, and P. Wyder, Phys. Rev. Lett. 79, 3238 (1997).

${ }^{10}$ M. R. Schaapman, P. C. M. Christianen, J. C. Maan, D. Reuter, and A. D. Wieck, Appl. Phys. Lett. 81, 1041 (2002).

${ }^{11}$ I. Meinel, D. Grundler, S. Bargstaedt-Franke, C. Heyn, D. Heitmann, and B. David, Appl. Phys. Lett. 70, 3305 (1997).

${ }^{12}$ D. Grundler, R. Eckart, B. David, and O. Dössel, Appl. Phys. Lett. 62, 2134 (1993).

${ }^{13}$ M. A. Wilde, M. Rhode, C. Heyn, D. Heitmann, D. Grundler, U. 
Zeitler, F. Schaffler, and R. J. Haug, Phys. Rev. B 72, 165429 (2005)

${ }^{14}$ A. Usher and M. Elliott, J. Phys.: Condens. Matter 21, 103202 (2009).

${ }^{15}$ M. A. Wilde, J. I. Springborn, O. Roesler, N. Ruhe, M. P. Schwarz, D. Heitmann, and D. Grundler, Phys. Status Solidi B 245, 344 (2008).

${ }^{16}$ S. Adachi, J. Appl. Phys. 58, R1 (1985).

${ }^{17}$ S. Dasgupta, C. Knaak, J. Moser, M. Bichler, S. F. Roth, A. Fontcuberta i Morral, G. Abstreiter, and M. Grayson, Appl. Phys. Lett. 91, 142120 (2007).

${ }^{18}$ S. Dasgupta, S. Birner, C. Knaak, M. Bichler, A. Fontcuberta i Morral, G. Abstreiter, and M. Grayson, Appl. Phys. Lett. 93, 132102 (2008).

${ }^{19}$ S. Dasgupta, Ph.D. thesis, Walter Schottky Institut, Technische Universität München, 2008.

${ }^{20}$ H. W. van Kesteren, E. C. Cosman, W. A. J. A. van der Poel, and C. T. Foxon, Phys. Rev. B 41, 5283 (1990).

${ }^{21}$ M. P. Schwarz, D. Grundler, I. Meinel, C. Heyn, and D. Heitmann, Appl. Phys. Lett. 76, 3564 (2000).

${ }^{22}$ M. A. Wilde, M. P. Schwarz, C. Heyn, D. Heitmann, D. Grundler, D. Reuter, and A. D. Wieck, Phys. Rev. B 73, 125325 (2006).

${ }^{23}$ M. A. Wilde, D. Reuter, C. Heyn, A. D. Wieck, and D. Grundler, Phys. Rev. B 79, 125330 (2009).

${ }^{24}$ T. Gokmen, M. Padmanabhan, O. Gunawan, Y. P. Shkolnikov, K. Vakili, E. P. De Poortere, and M. Shayegan, Phys. Rev. B 78,
233306 (2008).

${ }^{25}$ M. Zhu, A. Usher, A. J. Matthews, A. Potts, M. Elliott, W. G. Herrenden-Harker, D. A. Ritchie, and M. Y. Simmons, Phys. Rev. B 67, 155329 (2003).

${ }^{26}$ M. P. Schwarz, M. A. Wilde, S. Groth, D. Grundler, C. Heyn, and D. Heitmann, Phys. Rev. B 65, 245315 (2002).

${ }^{27}$ N. Ruhe, G. Stracke, C. Heyn, D. Heitmann, H. Hardtdegen, T. Schäpers, B. Rupprecht, M. A. Wilde, and D. Grundler, Phys. Rev. B 80, 115336 (2009).

${ }^{28}$ A. H. MacDonald, H. C. A. Oji, and K. L. Liu, Phys. Rev. B 34, 2681 (1986).

${ }^{29}$ I. M. A. Bominaar-Silkens, M. R. Schaapman, U. Zeitler, P. C. M. Christianen, J. C. Maan, D. Reuter, A. D. Wieck, D. Schuh, and M. Bichler, New J. Phys. 8, 315 (2006).

${ }^{30}$ L. Brey, N. F. Johnson, and B. I. Halperin, Phys. Rev. B 40, 10647 (1989).

${ }^{31}$ S. J. Papadakis, E. P. De Poortere, and M. Shayegan, Phys. Rev. B 59, R12743 (1999).

${ }^{32}$ K. Lai, W. Pan, D. C. Tsui, S. Lyon, M. Mühlberger, and F. Schäffler, Phys. Rev. Lett. 96, 076805 (2006).

${ }^{33}$ The simulator software nextnano ${ }^{3}$ can be obtained from http:// www.wsi.tum.de/nextnano3.

${ }^{34}$ A. Schmeller, J. P. Eisenstein, L. N. Pfeiffer, and K. W. West, Phys. Rev. Lett. 75, 4290 (1995).

${ }^{35}$ T. Ando and Y. Uemura, J. Phys. Soc. Jpn. 37, 1044 (1974).

${ }^{36}$ R. J. Nicholas, R. J. Haug, K. v. Klitzing, and G. Weimann, Phys. Rev. B 37, 1294 (1988). 